reporting unreliable. The aim of our study is to report outcomes from a prospective SENTIX trial that have implications for the standardisation of LLL assessment.

Methodology In the prospective international multicentre trial SENTIX (ENGOT-cx2/CEEGOG CX-01), a group of 150 patients with stage IA1-IB2 cervical cancer treated by uterine surgery with bilateral SLN biopsy was prospectively evaluated by objective LLL assessment, based on limb volume change (LVC) using circumferrential limb measurements and subjective patient-reported swelling. The assessments were conducted in six-month periods over 24 months post-surgery.

Result(s)* Patient LVC substantially fluctuated in both positive and negative directions (figure 1), which were comparable in frequency up to $14 \%+/$ - LVC increments. Thirty-eight patients experienced persistent LVC increase and $>10 \%$ classified as LLL, for whom median time to onset was nine months (95\% CI: 7.0-11.0). Some 34.2\% of cases experienced onset later than one year after the surgery. Thirty-three patients $(22 \%)$ experienced transient oedema characterised as LVC $>10 \%$, which resolved without intervention between two consequent follow-up visits (figure 2). No significant correlation between LVC $>10 \%$ and a patient-reported swelling was observed.

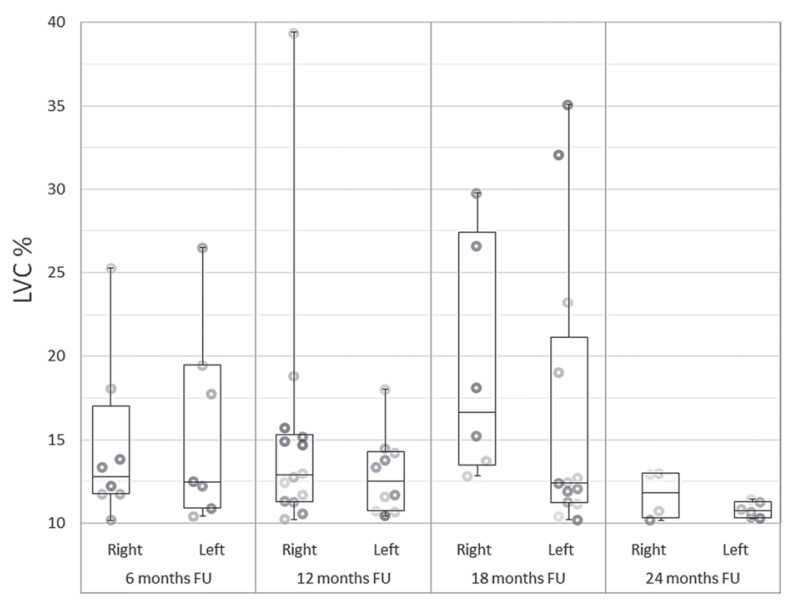

Abstract 959 Figure 2 Incidence of transient oedema. Each dot marks the transient oedema ( $>10 \%$ LVC increase from preoperative measurement) of one patient. Boxplots depict the median value of respective transient oedema $\mathrm{LVI}(\%)$; the percentile range was set at $25 \%$ and $50 \%$; the whisker is between minimal and maximal calculated value. FU; follow-up; LVC: limb volume change.

Conclusion* Our study showed that lower-limb volumes after surgical treatment of cervical cancer significantly fluctuate in positive and negative directions. A diagnostic threshold for LLL should be increased to $>15 \%$ LVC. Transient oedema occurs frequently, and its distinction from persistent LLL requires repeated measurements. One-third of new LLL cases were diagnosed in the second year of follow-up, highlighting the importance of a sufficient follow-up period duration. Finally, patient-reported limb swelling correlated poorly with LVC and should only be used as an adjunct to objective LLL assessment.

\section{THE ANNUAL RECURRENCE RISK MODEL FOR TAILORED SURVEILLANCE STRATEGY IN CERVICAL CANCER PATIENTS}

${ }^{1 ; 2}{ }^{2}$ Cibula*, ${ }^{3}$ J Jarkovsky, ${ }^{4} \mathrm{~L}$ Van Lonkhuijzen, ${ }^{5} \mathrm{H}$ Falconer, ${ }^{6} \mathrm{~A}$ Fagotti, ${ }^{7} \mathrm{~A}$ Ayhan, ${ }^{8} \mathrm{~S}$ Kim, ${ }^{9} \mathrm{D}$ Isla Ortiz, ${ }^{10} \mathrm{~J}$ Klat, ${ }^{11} \mathrm{~A}$ Obermair, ${ }^{12} \mathrm{~F}$ Landoni, ${ }^{13} \mathrm{~J}$ Rodriguez, ${ }^{14} \mathrm{R}$ Manchanda, ${ }^{15}$ K Kostun, ${ }^{16} \mathrm{R}$ Dos Reis, ${ }^{17} \mathrm{MM}$ Meydanli, ${ }^{18} \mathrm{D}$ Odetto, ${ }^{19} \mathrm{R}$ Laky, ${ }^{1 ; 2} \mathrm{M}$ Borčinová, ${ }^{20} \mathrm{~A}$ Lopez. ${ }^{1}$ First Faculty of Medicine, Charles University and General University Hospital, Prague, Czech Republic, Gynecologic Oncology Center, Department of Obstetrics and Gynecology, Prague, Czech Republic; ${ }^{2}$ Central and Eastern European Gynecologic Oncology Group, CEEGOG, Prague, Czech Republic; ${ }^{3}$ Institute of Biostatistics and Analyses, Faculty of Medicine, Masaryk University, Brno, Czech Republic; ${ }^{4}$ Amsterdam UMC, locatie AMC, Gynecologic Oncology, Amsterdam, Netherlands; ${ }^{5}$ Karolinska University Hospital and Department of Women's and Children's Health, Karolinska Institutet, Department of Pelvic Cancer, Stockholm, Sweden; ${ }^{6}$ Fondazione Policlinico Universitario A. Gemelli, IRCCS, UOC Ginecologia Oncologica, Dipartimento per la salute della Donna e del Bambino e della Salute Pubblica, Roma, Italy; ${ }^{7}$ Baskent University School of Medicine Department of Gynecology and Obstetrics Division of Gynecologic Oncology, Ankara, Turkey; ${ }^{8}$ Memorial Sloan Kettering Cancer Center, Department of Surgery, New York, USA; ${ }^{9}$ Gynecology Oncology Center, National Institute of Cancerology Mexico, Mexico, Mexico; ${ }^{10}$ Faculty of Medicine, University Hospital and University of Ostrava, Department of Obstetrics and Gynecology, Ostrava, Czech Republic; ${ }^{11}$ Queensland Centre for Gynaecological Cancer, The University of Queensland, Australia; ${ }^{12}$ University of Milano-Bicocca, Department of Obstetrics and Gynecology, Gynaecologic Oncology Surgical Unit, ASST-Monza, San Gerardo Hospital, Monza, Italy; ${ }^{13}$ Instituto Nacional de Cancerología, in Bogotá, Department of Gynecologic Oncology, Bogota, Colombia; ${ }^{14}$ Wolfson Institute of Preventive Medicine, Barts Cancer Centre, Queen Mary University of London, and Barts Health NHS Trust, London, UKi ${ }^{15}$ University Hospital Pilsen, Charles University in Prague, Department of Gynaecology and Obstetrics, Czech Republic; ${ }^{16}$ The University of Texas MD Anderson Cancer Center, Department of Gynecologic Oncology and Reproductive Medicine, Houston, USA; ${ }^{17} Z e k a i$ Tahir Burak Women's Health and Research Hospital, University of Health Sciences, Department of Gynecologic Oncology, Ankara, Turkey: ${ }^{18}$ Hospital Italiano de Buenos Aires, Instituto Universitario Hospital Italiano, Department of Gynecologic Oncology, Buenos Aires, Argentina; ${ }^{19}$ Medical University of Graz, Gynecology, Graz, Austria; ${ }^{20}$ National Institute of Neoplastic Diseases, Department of Gynecological Surgery, Lima, Peru

\subsection{6/ijgc-2021-ESG0.82}

Introduction/Background* Current guidelines for surveillance strategy in cervical cancer are rigid, recommending the same strategy for all survivors. The aim of this study was to develop a robust and comprehensive model allowing for individualised surveillance strategy based on risk profile of earlystage cervical cancer patients that were referred for surgical treatment with curative intent.

Methodology The data of 4,343 cervical cancer patients with pathologically confirmed early-stage cervical cancer treated between 2007 and 2016 were obtained from SCANN consortium centres of excellence (Surveillance in Cervical CANcer). Only patients with complete key predictor variables and a minimum of one-year follow-up data availability were included. Based on the prognostic markers, a multivariable Cox proportional hazards model predicting disease-free survival (DFS) was developed and internally validated. A risk score, derived from regression coefficients of the model, stratified the cohort into significantly distinctive risk groups. On its basis, the annual recurrence risk model (ARRM) was calculated by conditional survival analysis.

Result(s)* Five variables significant in multivariable analysis of recurrence risk were included in the prognostic model: maximal pathologic tumour diameter, tumour histotype, tumour grade, the number of positive pelvic lymph nodes, and lymphovascular space invasion (table 1). The model was ten-fold 
internally cross-validated with the average AUC of 0.732. Five risk groups significantly differing in prognosis were identified: with five-year DFS of $97.5 \%, 94.7 \%, 85.2 \%$, and $63.3 \%$ in consecutive increasing risk groups, while two-year DFS in the highest risk group equalled $15.4 \%$. Based on ARRM, the annual recurrence risk in the lowest risk group was below $1 \%$ in the first year of follow-up and declined below 1\% at years three, four, and $>5$ in the three medium-risk groups (figure 1 ). The proportion of pelvic recurrences declined in groups with the growing risk. In the whole cohort, $26 \%$ of

Abstract 960 Table 1 Multivariate model for risk of recurrence prediction

\begin{tabular}{|c|c|c|c|c|c|c|}
\hline Predictor & & $\beta$ & SE(B) & HR (95\% Cl) & $\boldsymbol{P}$-value & $\begin{array}{c}\text { Risk } \\
\text { points } \\
\text { (max. 100) }\end{array}$ \\
\hline \multirow[t]{5}{*}{ Histotype } & Squamous cell & & & Reference & & 0 \\
\hline & Adenocancer & 0.342 & 0.116 & $1.408(1.120 ; 1.771)$ & 0.003 & 7 \\
\hline & Adenosquamous & 0.598 & 0.164 & $1.819(1.317 ; 2.513)$ & $<0.001$ & 11 \\
\hline & Neuroendocrine & 1.741 & 0.246 & $5.704(3.514 ; 9.260)$ & $<0.001$ & 33 \\
\hline & Other & 1.145 & 0.270 & $3.144(1.848 ; 5.349)$ & $<0.001$ & 22 \\
\hline \multirow[t]{4}{*}{ Tumour diameter } & $<0.5 \mathrm{~cm}$ & & & Reference & & 0 \\
\hline & $0.5-1.99 \mathrm{~cm}$ & 0.501 & 0.237 & 1.651 (1.035; 2.634) & 0.035 & 10 \\
\hline & $2-3.99 \mathrm{~cm}$ & 1.115 & 0.236 & $3.051(1.915 ; 4.858)$ & $<0.001$ & 21 \\
\hline & $\geq 4 \mathrm{~cm}$ & 1.556 & 0.245 & $4.738(2.925 ; 7.674)$ & $<0.001$ & 30 \\
\hline \multirow[t]{3}{*}{ Grade } & 1 & & & Reference & & 0 \\
\hline & 2 & 0.260 & 0.214 & $1.297(0.852 ; 1.976)$ & 0.235 & 5 \\
\hline & 3 & 0.457 & 0.247 & $1.579(0.970 ; 2.570)$ & 0.085 & 9 \\
\hline \multirow[t]{4}{*}{ Positive pelvic LN } & $0 /$ not assessed & & & Reference & & 0 \\
\hline & 1 & 0.255 & 0.154 & $1.291(0.953 ; 1.748)$ & 0.098 & 5 \\
\hline & 2 & 0.482 & 0.170 & $1.619(1.158 ; 2.264)$ & 0.005 & 9 \\
\hline & $\geq 3$ & 0.939 & 0.144 & $2.557(1.927 ; 3.394)$ & $<0.001$ & 18 \\
\hline \multirow[t]{2}{*}{ LVSI } & No / not assessed & & & Reference & & 0 \\
\hline & Yes & 0.538 & 0.106 & $1.713(1.390 ; 2.111)$ & $<0.001$ & 10 \\
\hline
\end{tabular}

B: beta coefficient; Cl: confidence interval; LN: lymph node; LVSI: lymphovascular space invasion

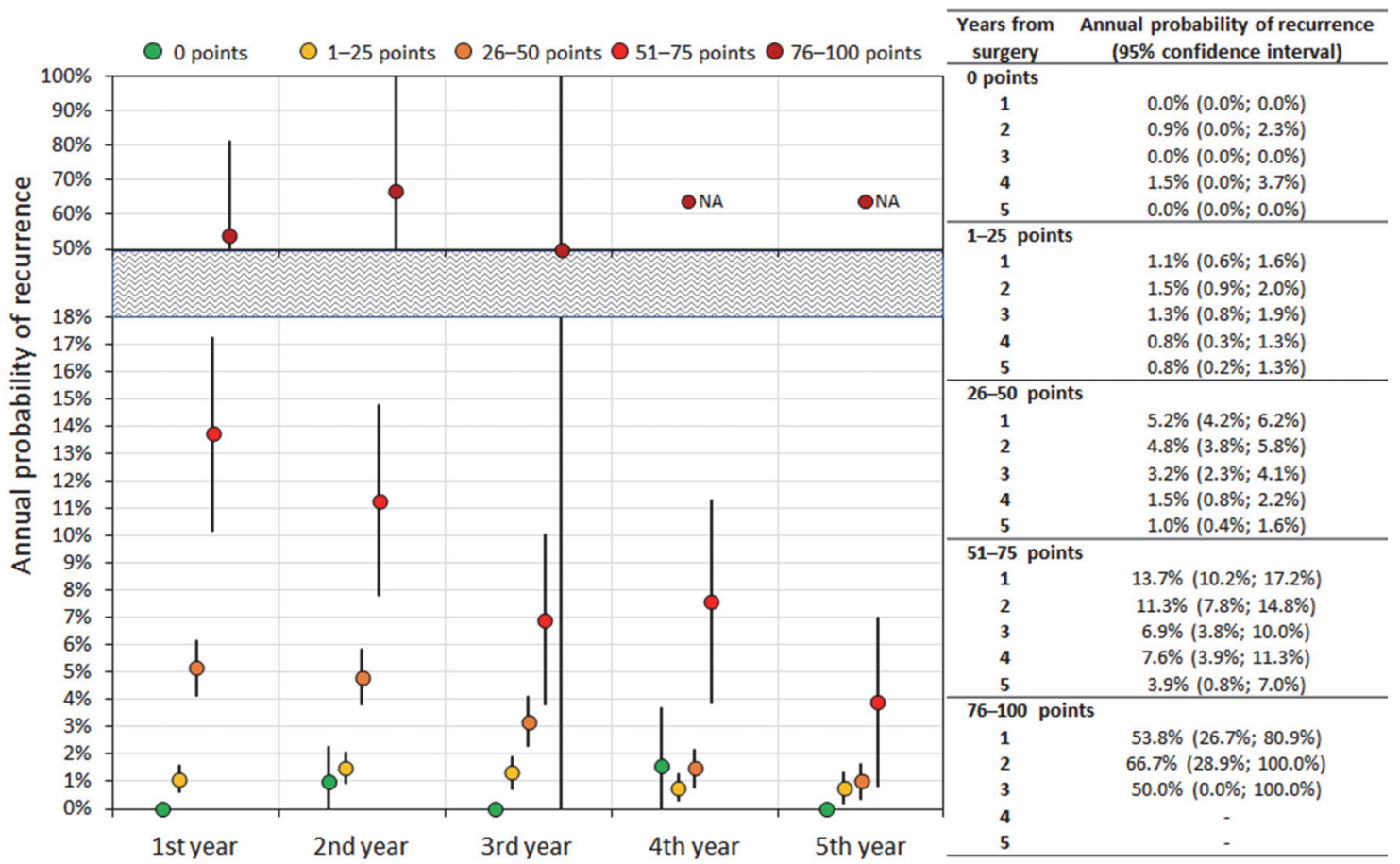

Abstract 960 Figure 1 ARRM: Landmark analysis of the annual probability of recurrence after surgery. N/A not analysed. 
recurrences appeared at the first year of the follow-up, $48 \%$ by year two, and $78 \%$ by year five.

Conclusion* ARRM represents a powerful tool for tailoring the surveillance strategy in early-stage cervical cancer patients based on the patient's risk status and respective annual recurrence risk. It can easily be utilised in routine clinical settings internationally.

\section{SENTIX - ACCURACY OF PREOPERATIVE LOCAL STAGING IN THE SENTIX TRIAL (CEEGOG-CX01; ENGOT- CX2; NCT02494063)}

IJ Klat*, ${ }^{2} \mathrm{R}$ Kocian, ${ }^{3} \mathrm{C}$ Kohler, ${ }^{4} \mathrm{~J}$ Jarkovsky, I Zapardiel, ${ }^{6} \mathrm{~A}$ Buda, ${ }^{7} \mathrm{~L}$ Van Lonkhuijzen, ${ }^{8} \mathrm{~B}$ Sehnal, ${ }^{9} \mathrm{O}$ Arencibia Sanchez, ${ }^{10} \mathrm{~A}$ Torne, ${ }^{11} \mathrm{~F}$ Raspagliesi, ${ }^{12} \mathrm{~J}$ Presl, ${ }^{13} \mathrm{M}$ Felsinger, ${ }^{14} \mathrm{R}$ Pilka, ${ }^{15} \mathrm{M}$ Ostojich, ${ }^{16} \mathrm{~A}$ Petiz, ${ }^{17} \mathrm{~S}$ Smrkolj, ${ }^{18} \mathrm{~F}$ Kridelka, ${ }^{19} \mathrm{P}$ Dundr, ${ }^{2} \mathrm{D}$ Cibula. 'University Hospital Ostrava, CEEGOG*, Department of Obstetrics and Gynecology, Ostrava, Czech Republic; ${ }^{2}$ First Faculty of Medicine, Charles University and General University Hospital in Prague, Prague, , CEEGOG*, Gynecologic Oncology Center, Department of Obstetrics and Gynecolog , Prague, Czech Republic; ${ }^{3}$ Asklepios-Clinic Hamburg, Department of Special Operative and Oncologic Gynaecology, Hamburg, Germany; ${ }^{4}$ Faculty of Medicine, Masaryk University, Institute of Biostatistics and Analyses, Brno, Czech Republic; ${ }^{5} \mathrm{La}$ Paz University Hospital, Department of Obstetrics and Gynecology, , Madrid, Spain; ${ }^{6}$ San Gerardo Hospital, Department of Obstetrics and Gynecology, Unit of Gynecologic Oncology Surgery, Monza, Italy; ${ }^{7}$ Academic Medical Centre, Center for Gynecologic Oncology, , Amsterdam, Netherlands; ${ }^{8}$ University Hospital Bulovka, First Faculty of Medicine, Charles University, CEEGOG*, Department of Obstetrics and Gynecology, Prague, Czech Republic; ${ }^{9}$ University Hospital of the Canary Islands, Department of Gynecologic Oncology, Las Palmas de Gran Canaria, Spain; ${ }^{10}$ Institute Clinic of Gynecology, Obstetrics and Neonatology (ICGON), Hospital Clinic of Barcelona, Unit of Gynecological Oncology, Barcelona, Spain: ${ }^{11}$ IRCCS Foundation National Cancer Institute in Milan, Milano, Italy; ${ }^{12}$ University Hospital Pilsen, Charles University, CEEGOG*, Department of Obstetrics and Gynecology, Pilsen, Czech Republic; ${ }^{13}$ Faculty of Medicine, Masaryk University, CEEGOG*, Department of Obstetrics and Gynecology, Brno, Czech Republic; ${ }^{14}$ Faculty of Medicine and Dentistry, Palacky University, University Hospital Olomouc, CEEGOG*, Department of Obstetrics and Gynecology, Olomouc, Czech Republic; ${ }^{15}$ Institute of Oncology Angel H. Roffo, University of Buenos Aires, Department of Gynaecology and Obstetrics, , Buenos Aires, Argentina; ${ }^{16}$ Francisco Gentil Portuguese Oncology Institute, Department of Gynecology, , Porto, Portugal; ${ }^{17}$ University Medical Centre Ljubljana, CEEGOG*, Ljubljana, Slovenia; ${ }^{18} \mathrm{CHU}$ Liege, Department of Obstetrics and Gynecology, Liege, Belgium; ${ }^{19}$ First Faculty of Medicine, Charles University and General University Hospital, Institute of Pathology, , Prague, Czech Republic

\subsection{6/ijgc-2021-ESG0.83}

Introduction/Background* The SENTIX is a prospective cohort international study on sentinel lymph node (SLN) biopsy without pelvic lymph node dissection (PLND) in patients with early-stage cervical cancer. The primary end point is a recurrence rate at 24 months' follow-up after the surgery. Either magnetic resonance imaging (MRI) or expert ultrasound (EUS) was mandatory as a preoperative staging method. The aim of this study is to report the accuracy of preoperative local staging.

Methodology Forty-seven sites from 18 countries participated in the study. Patients with stages T1a1/LVSI+ - T1b1 (FIGO 2009), common histological types and no suspicious lymph nodes on imaging were eligible. Patients were excluded from further study if SLN were not detected on both sides and if SLN was positive on frozen section histological evaluation. Compared were results from preoperative imaging with final pathology reports.

Result(s)* From May 2016 to October 2020, 733 registered patients underwent surgery, 132 were excluded intraoperatively, data from 708 were analysed in this study. Patients' characteristics are in table 1 . Out of 90 patients clinically

\section{Tumour stage:}

\section{Upstaging}
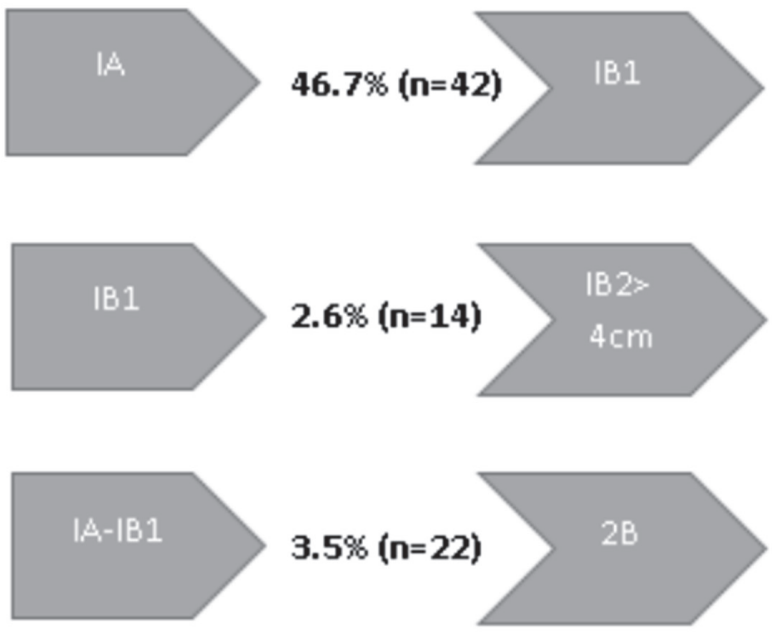

\section{Downstaging}

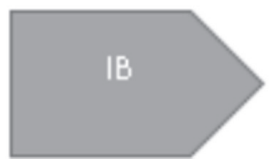

\section{$6.0 \%(n=33)$}



Abstract 966 Figure 1 Chart 1 the accuracy of local staging

Abstract 966 Table 1 Patient's characteristics

\begin{tabular}{|c|c|c|}
\hline \multicolumn{2}{|l|}{ Parameter } & \multirow{2}{*}{$\begin{array}{c}\mathrm{N}(\%) / \text { me dian (5-95 th } \\
\text { percentile) }\end{array}$} \\
\hline Age & & \\
\hline & $\leq 40$ & $294(40.1 \%)$ \\
\hline & $41-60$ & $339(46.2 \%)$ \\
\hline & $61+$ & $100(13.6 \%)$ \\
\hline \multirow[t]{5}{*}{ BMI } & $\leq 20$ & $73(10.0 \%)$ \\
\hline & $20-25$ & $345(47.1 \%)$ \\
\hline & $25-30$ & $169(23.1 \%)$ \\
\hline & $30+$ & $141(19.2 \%)$ \\
\hline & NA & $5(0.7 \%)$ \\
\hline \multirow[t]{3}{*}{ ECOGPS } & 0 & $701(95.6 \%)$ \\
\hline & 1 & $28(3.8 \%)$ \\
\hline & NA & $4(0.6 \%)$ \\
\hline \multirow[t]{3}{*}{ Diagnostic method } & Biopsy & $331(45.2 \%)$ \\
\hline & Conization & $399(54.4 \%)$ \\
\hline & NA & $3(0.4 \%)$ \\
\hline \multicolumn{3}{|l|}{ Imaging: } \\
\hline EUS & Yes & $392(53.5 \%)$ \\
\hline MRI & Yes & $411(56.1 \%)$ \\
\hline \multirow{2}{*}{$\begin{array}{l}\text { Maximum pre ope rative } \\
\text { tumour size (mm) }\end{array}$} & $\leq 20$ & $471(64.2 \%)$ \\
\hline & $20.1-40$ & $262(35.8 \%)$ \\
\hline \multirow[t]{4}{*}{ Pre ope rative tumour stage } & $1 \mathrm{~A} 1$ & $65(8.9 \%)$ \\
\hline & $1 \mathrm{~A} 2$ & $32(4.4 \%)$ \\
\hline & $1 \mathrm{~B} 1 \leq 2 \mathrm{~cm}$ & $374(51.0 \%)$ \\
\hline & $1 \mathrm{~B} 1>2 \mathrm{~cm}$ & $262(35.7 \%)$ \\
\hline \multirow[t]{4}{*}{ Tumour grade } & G1 & $179(24.4 \%)$ \\
\hline & G2 & $373(50.9 \%)$ \\
\hline & G3 & $157(21.4 \%)$ \\
\hline & NA & $24(3.3 \%)$ \\
\hline \multirow[t]{4}{*}{ Tumour type } & $\mathrm{scC}$ & $508(69.3 \%)$ \\
\hline & $A C$ & $210(28.6 \%)$ \\
\hline & AS & $9(1.2 \%)$ \\
\hline & NA & $6(0.8 \%)$ \\
\hline Screening failure (SF): & & $131(17.7 \%)$ \\
\hline \multirow[t]{2}{*}{ Preoperatively } & Surgery cancelled & $4(0.5 \%)$ \\
\hline & ICF withdrawn & $4(0.5 \%)$ \\
\hline \multirow{3}{*}{ Intraoperatively } & SLN not detected bilaterally & $55(7.5 \%)$ \\
\hline & Me tastatic SLN involvement & $48(6.5 \%)$ \\
\hline & $>1 \mathrm{B1}$ & $12(1.6 \%)$ \\
\hline Other & & $8(1.1 \%)$ \\
\hline
\end{tabular}

